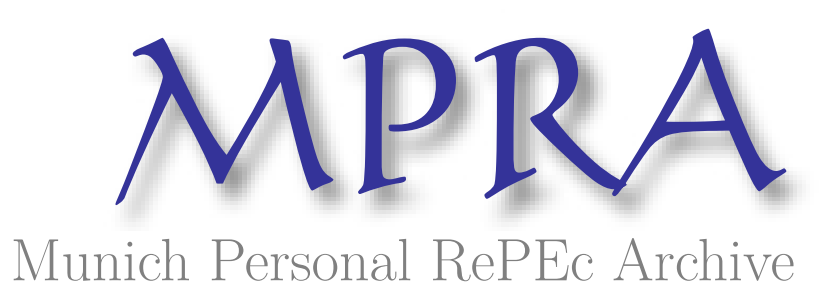

\title{
Multi-Outcome Lotteries: Prospect Theory vs. Relative Utility
}

Kontek, Krzysztof

Artal Investments

28 May 2010

Online at https://mpra.ub.uni-muenchen.de/22947/

MPRA Paper No. 22947, posted 30 May 2010 06:35 UTC 


\title{
Multi-Outcome Lotteries: \\ Prospect Theory vs. Relative Utility
}

\author{
Krzysztof Kontek ${ }^{1}$ \\ Artal Investments, Warsaw ${ }^{2}$
}

\begin{abstract}
This paper discusses two approaches for the analysis of multi-outcome lotteries. The first uses Cumulative Prospect Theory. The second is the Relative Utility Function, which strongly resembles the utility function hypothesized by Markowitz (1952). It is shown that the relative utility model follows Expected Utility Theory with a transformed outcome domain. An illustrative example demonstrates that not only it is a simpler model, but it also provides more sound predictions regarding certainty equivalents of multi-outcome lotteries. The paper discusses estimation procedures for both models. It is noted that Cumulative Prospect Theory has been derived using two-outcome lotteries only, and it is hard to find any evidence in the literature of its parameters ever having been estimated by using lotteries with more than two outcomes. Least squares (mean) and quantile (including median) regression estimations are presented for the relative utility model. It turns out that the estimations for two- and threeoutcome lotteries are essentially the same. This confirms the correctness of the model and vindicates the homogeneity of responses given by subjects. An additional advantage of the relative utility model is that it allows multi-outcome lotteries, together with the estimation results, to be presented on a single graph. This is not possible using Cumulative Prospect Theory.
\end{abstract}

JEL classification: C13, C21, C51, C91, D03, D81, D87

Keywords: Multi-Prize Lotteries, Lottery / Prospect Valuation, Markowitz Hypothesis, Prospect / Cumulative Prospect Theory, Aspiration / Relative Utility Function.

\footnotetext{
${ }^{1}$ The author is grateful to Stefan Traub from the Department of Economics, University of Bremen and Ulrich Schmidt from the Department of Economics, University of Kiel for making the results of their experiments available.

${ }^{2}$ Contact: ul. Chrościckiego 93/105, 02-414 Warsaw, Poland,

e-mail: kontek@artal.com.pl, kkontek2000@yahoo.com, tel.: +48 504161750.
} 


\section{Introduction}

1.1. One of the main objections to the original version of Prospect Theory (Kahneman, Tversky, 1979) is that it can only handle two-outcome lotteries. This defect was remedied by Cumulative Prospect Theory, which "employs cumulative rather than separable decision weights" (Tversky, Kahneman, 1992). This approach makes it possible to generalize the theory to multi-outcome lotteries.

Kontek (2009a) demonstrated that the same experimental data used to derive Cumulative Prospect Theory can lead to a completely different solution, one which resembles the utility function hypothesized by Markowitz (1952). This solution was named the "relative utility" or "aspiration" function. In contrast to Prospect Theory, this is a single-equation model and does not require the concept of probability weighting to explain the experimental results. Kontek (2009b) has shown that the relative utility model may be applied to multioutcome lotteries as well and that the lottery valuation follows the Expected Utility approach with a transformed outcome domain.

1.2. This paper discusses both approaches for multi-outcome lotteries. Point 2 presents Cumulative Prospect Theory, whereas Point 3 is devoted to a demonstration of the relative utility function. Point 4 presents an illustrative example of a three-outcome lottery showing that both models lead to different solutions. This example demonstrates that not only is the relative utility model simpler, but that it also gives a more sound prediction of certainty equivalents in the case of multi-outcome lotteries. This prediction is, moreover, consistent with the experimental results for two-outcome lotteries.

Point 5 is devoted to estimating the model parameters using experimental data. It is first stated that the Cumulative Prospect Theory parameters were derived using two-outcome lotteries only (Tversky, Kahneman, 1992). More surprisingly, it is hard to find any evidence of multi-outcome lotteries being used to derive the parameters of the Cumulative Prospect Theory model in the literature ${ }^{3}$. This is quite an astonishing finding, especially given that it was made 18 years after the introduction of Cumulative Prospect Theory and 8 years after Daniel Kahneman being awarded the Nobel Prize for this theory. Cumulative Prospect Theory would appear to be more a mathematical model rather than a theory confirmed by conducted experiments.

Point 5 presents an estimation of the relative utility model using experimental data by

\footnotetext{
${ }^{3}$ To the best of the knowledge of the author of this paper and that of a few other more experienced researchers, who were asked by the author for help.
} 
Schmidt and Traub (2009). These data include both two- and three-outcome lotteries. The presented estimations are essentially the same in both cases. This not only proves the correctness of the relative utility model, it also confirms that people's decisions are homogeneous, i.e. they are not influenced by the number of outcomes.

Mean (least squares) and quantile regression estimations for the relative utility model are presented in Point 5. This confirms the attractiveness of the model from the estimation point of view. This has already been stated in previous papers by Kontek (2010a, 2010b) for two-outcome lotteries. By contrast, almost every derivation of the Prospect Theory model encountered in the literature involves the least squares procedure.

An additional advantage of the relative utility model is that it allows multi-outcome lotteries to be represented together with the estimation results on a single graph. This is not possible using Cumulative Prospect Theory. This topic is discussed in Point 6.

Point 7 summarizes the paper with the conclusion that the relative utility model offers a number of advantages over Cumulative Prospect Theory in regard to multi-outcome lotteries.

\section{Cumulative Prospect Theory}

2.1. Let us assume that a set of outcomes $X$ partitions the possible states of the world into mutually exclusive events. Let us then define a vector of lottery outcomes $x=\left\{x_{1}, x_{2}, \ldots\right.$, $\left.x_{n}\right\}$ such that $x_{1}, x_{2}, \ldots, x_{n} \in X$, and a vector of associated lottery probabilities $p=\left\{p_{1}, p_{2}, \ldots\right.$, $\left.p_{n}\right\}$ such that $\sum_{i=1}^{n} p_{i}=1$, where $p_{i}$ is the probability of outcome $x_{i}$. A lottery $y$ is therefore defined as a pair of vectors $y=\{x, p\}$. The expected value of a lottery can be determined similarly to any random event:

$$
E X=\sum_{i=1}^{n} x_{i} p_{i}
$$

The sum in (2.1) can be replaced by the integral in case of continuous outcome distribution. This generalization, however, is not considered in this paper.

2.2. Assuming there exists a certain utility function $U$, let us determine an outcome utility vector $u=\left\{u_{1}, u_{2}, \ldots, u_{n}\right\}$, where $u_{i}=\mathrm{U}\left(x_{i}\right)$. This enables the Expected Utility valuation to be presented as:

$$
\mathrm{V}(y)=\sum_{i=1}^{n} \mathrm{U}\left(x_{i}\right) p_{i}=\sum_{i=1}^{n} u_{i} p_{i}
$$


2.3. Deviations from Expected Utility Theory (such as those observed in the Allais paradox) could be explained by people having a nonlinear perception of probabilities. This led to the concept of probability weighting presented by Edwards (1961). Kahneman and Tversky proposed the following formula for lottery valuation in their Prospect Theory (1979):

$$
\mathrm{V}(y)=\sum_{i=1}^{n} \mathrm{U}\left(x_{i}\right) \mathrm{W}\left(p_{i}\right)=\sum_{i=1}^{n} u_{i} w_{i}
$$

where $\mathrm{W}(p)$ is the probability weighting function, and $w_{i}=\mathrm{W}\left(p_{i}\right)$ are the weights assigned to specific probabilities and which form a vector of weighted probabilities $w=\left\{w_{1}, w_{2}, \ldots, w_{n}\right\}$.

2.4. Prospect Theory is incorrect from a mathematical point of view as (2.3) leads to a violation of the first order stochastic dominance when weights are assigned to single probabilities. A solution to this problem was proposed by Quiggin in 1982 in his Rank-Dependent Expected Utility Theory (which he called Anticipated Utility Theory). The procedure proposed by Quiggin was adopted virtually unchanged by Cumulative Prospect Theory (1992) and is therefore presented here in a greater detail.

Quiggin assumed that the probability weighting function does not relate to individual probabilities, but to their cumulative values. A probability vector $p=\left\{p_{1}, p_{2}, \ldots, p_{n}\right\}$ is first ordered by ranking the outcomes $x_{i}$ (e.g. $x_{1}>x_{2}>\ldots>x_{n}$ ). A cumulative probability vector $c p$ $=\left\{c p_{1}, c p_{2}, c p_{3}, \ldots, c p_{n}\right\}=\left\{p_{1}, p_{1}+p_{2}, p_{1}+p_{2}+p_{3}, \ldots, 1\right\}$ is then introduced. Next, by applying the probability weighting function $\mathrm{W}$, a weighted cumulative probability vector $w=$ $\left\{w_{1}, w_{2}, \ldots, 1\right\}$ is obtained where $w_{i}=\mathrm{W}\left(c p_{i}\right)$. Finally, a vector of weighted cumulative probability increments is defined such that $\Delta w=\left\{\Delta w_{1}, \Delta w_{2}, \ldots, \Delta w_{n}\right\}=\left\{w_{1}, w_{2}-w_{1}, w_{3}-w_{2}, \ldots, 1\right.$ $\left.-w_{n-1}\right\}$. This is now used to calculate the lottery value:

$$
\begin{aligned}
\mathrm{V}(y) & =\sum_{i=1}^{n} \mathrm{U}\left(x_{i}\right) \Delta\left(\mathrm{W}\left(\sum_{k=1}^{i} p_{k}\right)\right)=\sum_{i=1}^{n} \mathrm{U}\left(x_{i}\right) \Delta\left(\mathrm{W}\left(c p_{i}\right)\right) \\
& =\sum_{i=1}^{n} \mathrm{U}\left(x_{i}\right) \Delta\left(w_{i}\right)=\sum_{i=1}^{n} u_{i} \Delta w_{i} .
\end{aligned}
$$

It is obvious that the lottery value obtained using the proposed methodology depends on the order of the components in the $x$ and $p$ vectors. This is because the cumulative probability vector and any subsequent results depend on how the outcomes are ranked. It should be noted that this approach simplifies to the original Prospect Theory from 1979 for a lottery with two outcomes.

2.5. Cumulative Prospect Theory (Tversky, Kahneman, 1992) introduced some extensions to the presented procedure in regard to negative outcomes. More specifically, it allowed 
different weighting functions for gains and losses. For simplicity, however, negative outcomes are not considered in this paper.

Cumulative Prospect Theory, on the basis of experimental data, determined the shape of the utility function $\mathrm{U}(x)$ (which the theory calls the value function):

$$
\mathrm{v}(x)=\lambda|x|^{\alpha},
$$

and the probability weighting function $\mathrm{W}(p)$ :

$$
\mathrm{W}(p)=\frac{p^{\gamma}}{\left(p^{\gamma}+(1-p)^{\gamma}\right)^{1 / \gamma}},
$$

where $\lambda=1$ and $\gamma=0.61$ for positive prospects (lotteries), $\lambda=-2.25$ and $\gamma=0.69$ for negative prospects, and $\alpha=0.88$ is valid for both positive and negative prospects.

\section{Relative Utility Function}

3.1. By analyzing the same data used to derive Cumulative Prospect Theory, Kontek (2009a) came up with a completely different solution (Figure 3.1, left), one whose shape resembled the utility function proposed by Markowitz in his 1952 paper "The Utility of Wealth" (Figure 3.2, right). The obtained curve, which Kontek called the aspiration function, explains the results of the experiments conducted by Kahneman and Tversky without using a probability weighting function. In this paper, the function is called the relative utility function and only its part for gains is considered.
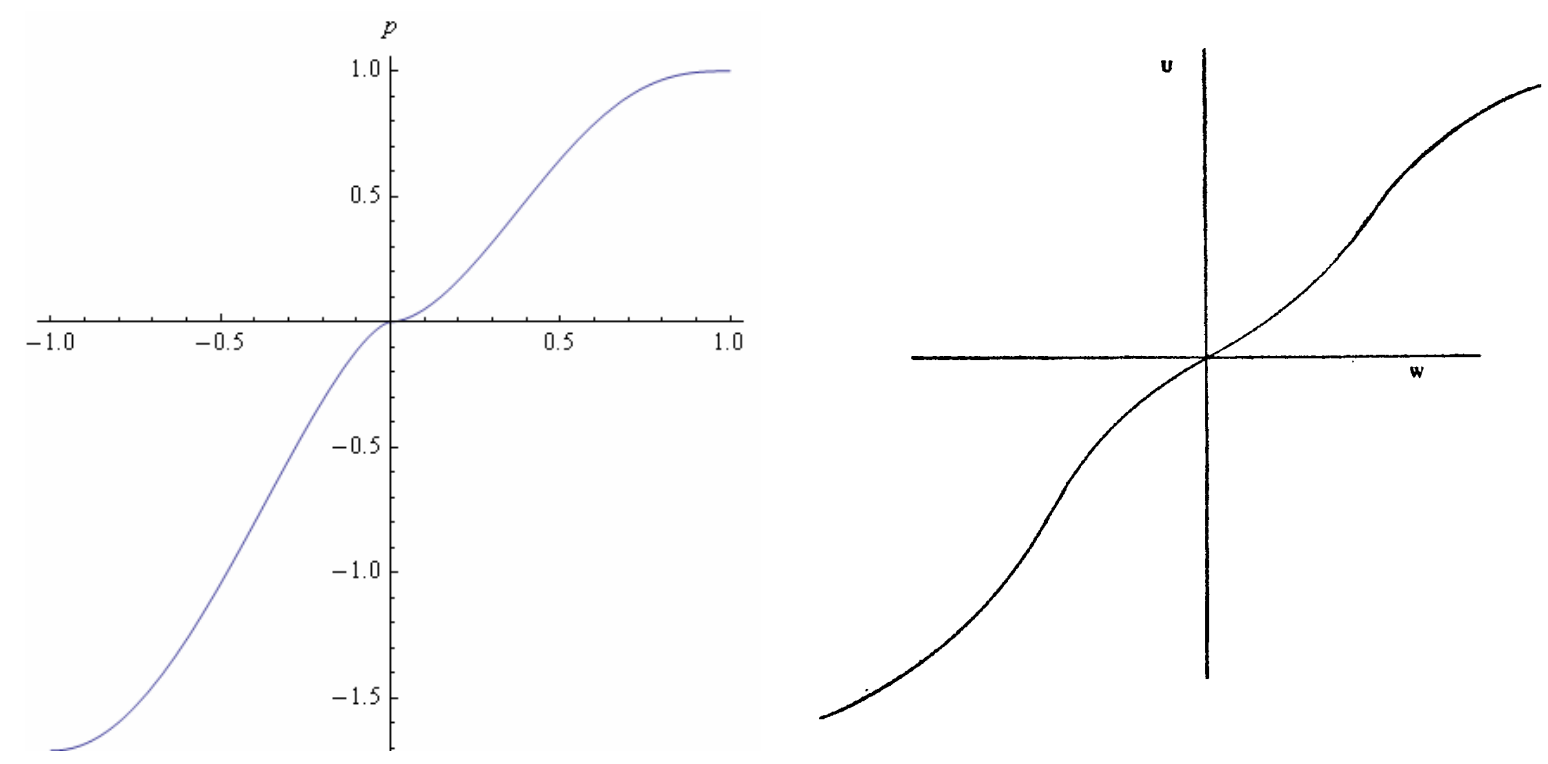

Figure. 3.1. Relative utility function $p=\mathrm{Q}(r)$ (Left); Utility function as presented in the Markowitz hypothesis (1952) (Right).

3.2. The relative utility model assumes a direct relationship between probability and 
the relative certainty equivalents for a two outcome lottery:

$$
p=\mathrm{Q}(r),
$$

where $p$ denotes probability, Q denotes a relative utility function, which should have the form of a cumulative density function defined over the range [0,1], and $r$ denotes the relative certainty equivalent defined as:

$$
r=\frac{c e-P_{\min }}{P_{\max }-P_{\min }}
$$

where $c e$ denotes the certainty equivalent, $P_{\max }=\operatorname{Max}(x)$ is the maximum lottery outcome (prize), and $P_{\min }=\operatorname{Min}(x)$ is the minimum lottery outcome (prize). The relationship described by (3.2) ensures that $r$ assumes values in the range $[0,1]$, even in the case of lotteries with a risk free $P_{\min }$ component. Equation (3.2) simplifies to $r=\frac{c e}{P_{\max }}$ for $P_{\min }=0$.

Knowing the relative certainty equivalent $r$, the certainty equivalent $c e$ of a lottery can be determined as:

$$
c e=P_{\min }+r\left(P_{\max }-P_{\min }\right)
$$

which, for $P_{\min }=0$, simplifies to $c e=r P_{\max }$.

As probability $p$ is a single-variable function of the relative certainty equivalent $r, r$ can be easily represented as a function of $p$ :

$$
r=\mathrm{Q}^{-1}(p)
$$

where $\mathrm{Q}^{-1}$ is the inverse form of the relative utility function. Because there are certainty equivalents which are typically determined in experiments rather than probabilities, the inverse form (3.4) of the relative utility function is more frequently used in estimation procedures.

3.3. Derivation of the model for multiple outcomes is straightforward. It is assumed that for each lottery with more than two outcomes in the range [0,1], there exists an equivalent lottery with two outcomes of 0 and 1 only. The probability of winning this equivalent lottery can be determined as follows. Each outcome $r_{i}$ of the multi-outcome lottery can be considered the certainty equivalent of a lottery, whose outcomes are 0 and 1 , and whose probability of winning is $p_{i}^{\prime}=\mathrm{Q}\left(r_{i}\right)$ (cf. (3.1)). The joint probability of winning the equivalent lottery is therefore $\sum_{i=1}^{n} p_{i} p_{i}^{\prime}$, which leads to: 


$$
p_{e q}=\sum_{i=1}^{n} p_{i} \mathrm{Q}\left(r_{i}\right)
$$

This $p_{e q}$ probability is called the equivalent probability of the multi-outcome lottery. The relative certainty equivalent of the multi-outcome lottery can be found by applying (3.4) to $p_{e q}$ :

$$
r=\mathrm{Q}^{-1}\left(\sum_{i=1}^{n} p_{i} \mathrm{Q}\left(r_{i}\right)\right)
$$

This ends the derivation.

3.4. Please note that (3.5) and (3.6) do not require the concept of probability weighting in either its basic or cumulative form. Nor do they require outcomes and their associated probabilities to be ranked. Finally, they are not impacted by representing a lottery outcome as several outcomes of the same value and of lower values of probability (which was a big problem in the original Prospect Theory version).

3.5. Please note the strong resemblance of the equivalent probability formula (3.5) to the Expected Utility valuation (2.2). In fact, the relative utility model follows Expected Utility Theory with a transformed outcome domain ${ }^{4}$. Relative utility is expressed in terms of probability (of winning the main prize or fulfilling aspirations) and does not require any hypothetical "utils" to describe people's behavior. To put it more straightforwardly, the equivalent probability is the relative utility. Once accustomed to this seemingly strange notion, everything can be considered at the basic probability theory level. This allows the relative utility model to avoid any complex topological concepts (like the Choquet integral) for its axiomatization, as is the case with Cumulative Prospect Theory.

\section{An Illustrative Example of a Three-Outcome Lottery}

4.1. We consider first a lottery with two outcomes: $\$ 0$ and $\$ 100$, each with a 0.5 probability of occurrence. As stated in Tversky and Kahneman's experiment (see Tab. 3.3 from their 1992 paper) the certainty equivalent of such a lottery was observed to be $\$ 36$. Next we

\footnotetext{
${ }^{4}$ It should be emphasized that transforming certainty equivalents to their relative form in the $[0,1]$ range is not just an artificial transformation. It is based first on the Weber Law, which is one of the fundamental laws of psychophysics, and second on mental transformations: Mental Adaptation and Attention Focus (Kontek, 2009a). These fundamental laws result in the relative rather than absolute notion of wealth changes. It follows that the certainty equivalents of a lottery are perceived in relation to its main prize. This observation is not new and is close in meaning to "mental accounting" (Thaler, 1980) or "framing" (Kahneman, Tversky, 1985). However Kahneman and Tversky did not include this observation in their Cumulative Prospect Theory in 1992. The relative notion of wealth changes makes the concept of probability weighting unnecessary to describe people's behavior and leads to a much simpler model, as presented.
} 
consider a second lottery having three outcomes: $\$ 0, \$ 36$ and $\$ 100$, with respective probabilities $(1-p) / 2, p$, and $(1-p) / 2$. The question concerns the certainty equivalent of this lottery.

It is noted that the outcome of $\$ 36$ is the same as the certainty equivalent of the first lottery. Both lotteries also have the same minimum and maximum outcomes, i.e. $\$ 0$ and $\$ 100$. In this case, the relative utility model assumes that people should be indifferent between the second lottery and a third one in which the outcome of $\$ 36$ is replaced by the first lottery. The third lottery clearly reduces to a two-outcome lottery as all outcomes are then $\$ 0$ or $\$ 100$. The probability of winning $\$ 100$ is expressed by:

$$
p_{e q}=p(1 / 2)+(1-p) / 2=1 / 2
$$

However, the certainty equivalent of a lottery with two outcomes $\$ 0$ and $\$ 100$, and a probability of 0.5 of winning the main prize, is $\$ 36$, as stated before. This means the certainty equivalent of the second lottery is $\$ 36$, which answers the posed question.

Please note that this result is independent of the relative utility shape; all that need be known is that it assumes a value of $0.36=\$ 36 / \$ 100$ for probability 0.5 . The result is also independent of the probability $p$ of the outcome $\$ 36$ in the second lottery. This is, however, a very logical conclusion. As $p$ approaches 0 , the second lottery reduces to the first lottery, whose certainty equivalent is $\$ 36$. As $p$ approaches 1 , the second lottery reduces to a sure payment of $\$ 36$. The second lottery may therefore be regarded as a mixture of two prospects having the same equivalent of $\$ 36$. Its certainty equivalent should therefore be $\$ 36$ whatever the $p$ value, i.e. however these prospects are mixed.

4.2. Let us now consider the same example using Cumulative Prospect Theory. According to this theory, the value of the second lottery is expressed as:

$$
\begin{aligned}
\mathrm{V}(y) & =\mathrm{v}(\$ 100)[\mathrm{W}((1-p) / 2)-\mathrm{W}(0)] \\
& +\mathrm{v}(\$ 36)[\mathrm{W}((1-p) / 2+p)-\mathrm{W}((1-p) / 2)] \\
& +\mathrm{v}(\$ 0)[\mathrm{W}(1)-\mathrm{W}((1-p) / 2+p)]
\end{aligned}
$$

where $\mathrm{v}$ and $\mathrm{W}$ are defined by (2.5) and (2.6). On the other hand, the value of the certainty equivalent $c e$ is equal to:

$$
\mathrm{V}(c e)=\mathrm{v}(c e) \mathrm{W}(1)
$$

Substituting (2.5) and (2.6) into (4.2) and (4.3) results, after simplification, in the following relationship: 


$$
\begin{aligned}
c e^{\alpha} & =100^{\alpha} \frac{((1-p) / 2)^{\gamma}}{\left[((1-p) / 2)^{\gamma}+((1+p) / 2)^{\gamma}\right]^{1 / \gamma}} \\
& +36^{\alpha} \frac{((1+p) / 2)^{\gamma}}{\left[((1-p) / 2)^{\gamma}+((1+p) / 2)^{\gamma}\right]^{1 / \gamma}} \\
& -36^{\alpha} \frac{((1-p) / 2)^{\gamma}}{\left[((1-p) / 2)^{\gamma}+((1+p) / 2)^{\gamma}\right]^{1 / \gamma}}
\end{aligned}
$$

where $\alpha$ and $\gamma$ are the model parameters given in point 3.5. This, after further reduction, leads to the solution for $c e$ :

$$
c e=\left\{\frac{\left(100^{\alpha}-36^{\alpha}\right)((1-p) / 2)^{\gamma}+36^{\alpha}((1+p) / 2)^{\gamma}}{\left[((1-p) / 2)^{\gamma}+((1+p) / 2)^{\gamma}\right]^{1 / \gamma}}\right\}^{\frac{1}{\alpha}}
$$

Obviously, this result can only be calculated numerically. Figure 4.1. presents this solution for all values of $p$ from 0 to 1 .

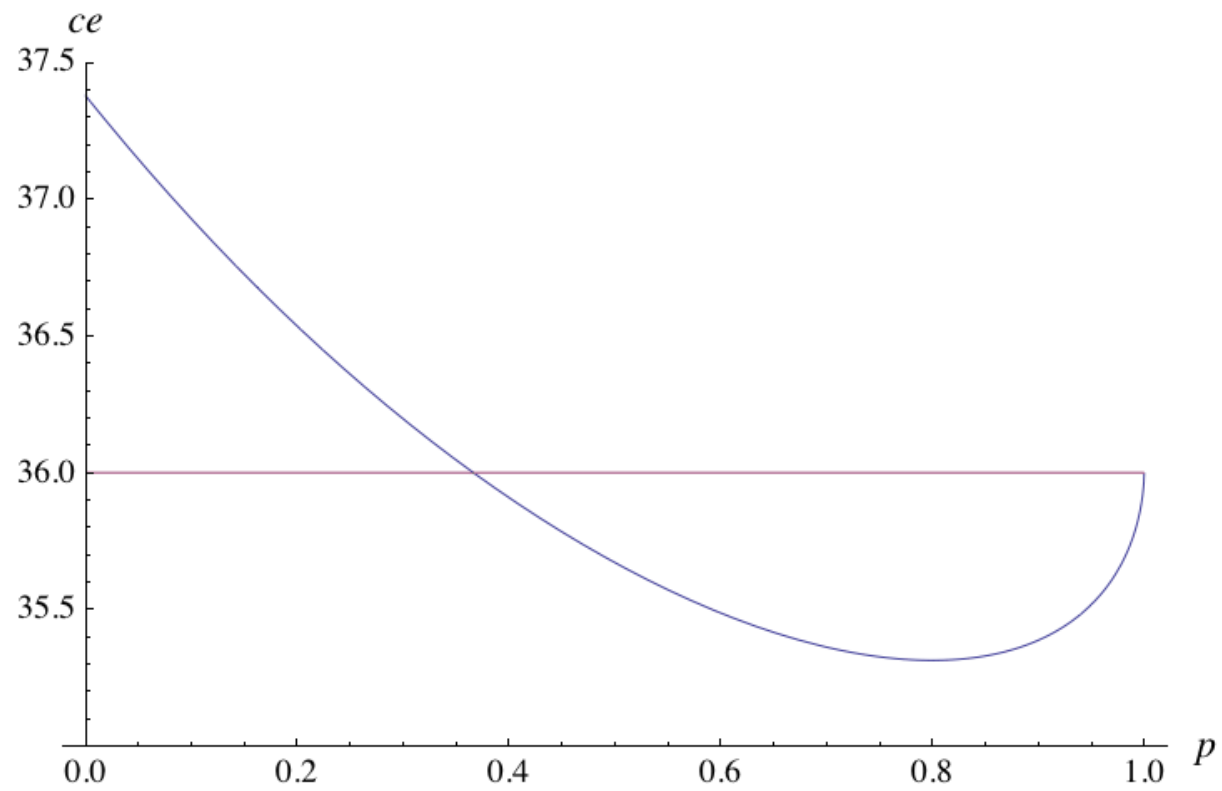

Figure 4.1. Certainty equivalent of a three-outcome lottery as a function of probability $p$ of a $\$ 36$ outcome. The solution for the relative utility model is a constant $\$ 36$. The solution for CPT is marked in blue.

The $c e$ value for the relative utility model is a constant $\$ 36$, as explained. In the case of Cumulative Prospect Theory, ce assumes a value of $\$ 36$ for $p=1$ (as expected) and a value of $\$ 37.4$ for $p=0$, which is inconsistent with the experimental data. The certainty equivalent assumes values greater than $\$ 36$ for probabilities lower than 0.366 and values lower than $\$ 36$ 
for probabilities greater than 0.366 . The certainty equivalent assumes a minimum value of $\$ 35.1$ for a probability of 0.8 . All these values clearly depend on the Prospect Theory parameterization.

4.3. The presented example demonstrates that the relative utility model is not only simpler but produces a more sound result, which, moreover, is consistent with the experimental data for two-outcome lotteries. In fact, the solution obtained using Cumulative Prospect Theory can hardly find any explanation and this task is left to the proponents of the theory.

\section{Model Estimation for Multi-Outcome Lotteries}

5.1. It is a frequently overlooked fact that the Cumulative Prospect Theory model parameters were derived using two-outcome lotteries only. The data used for this purpose are presented in Table 3.3. in Tversky and Kahneman's paper (1992) and consist of 28 twooutcome lotteries for gains and 28 two-outcome lotteries for losses. Applying the theory to multi-outcome lotteries has only been justified by a mathematical proof based on pretty complex topological concepts (like the Choquet integral) ${ }^{5}$. Nevertheless, it is commonly believed that this theory works for any number of outcomes. However, it is hard to find any evidence in the literature of the Cumulative Prospect Theory model ever having been estimated using lotteries with more than two outcomes. The only trace the author of this paper could find was in Gonzales and $\mathrm{Wu}$ (1999), who stated (footnote 6): "In addition to the two outcome gambles, 36 three-outcome gambles were included (conducted KK). Data from these gambles will be presented elsewhere". The author is not aware of any further work of Gonzales and Wu on this subject and is ignorant as to why these results, which would confirm the applicability of CPT to multi-outcome lotteries, have yet to be presented. That an experimental confirmation of this theory for multi-outcome lotteries should be lacking for so long is an extremely surprising finding, and one that calls the correctness and applicability of the theory into question.

5.2. One of the possibilities of this lack is that the Cumulative Prospect Theory model is very inconvenient for estimation purposes as it does not follow a classical econometric approach when building a regression model. In the case of Cumulative Prospect Theory, the following relationship for a two-outcome lottery has to be resolved using the estimation procedure:

$$
\mathrm{v}(c e)=\mathrm{v}\left(P_{\max }\right) \mathrm{W}\left(p_{1}\right)+\mathrm{v}\left(P_{\min }\right)\left[1-\mathrm{W}\left(p_{1}\right)\right]
$$

\footnotetext{
${ }^{5}$ This proof is given in the Appendix of the Tversky and Kahneman's paper, however it is not present in their selected works "Choices, Values, and Frames", Cambridge University Press, 2000. The author of the proof is P. Wakker.
} 
where $P_{\max }=\operatorname{Max}(x)$ is the maximum lottery outcome, $p$ denotes the probability of winning the prize, and the two functions $\mathrm{v}$ and $\mathrm{W}$ are estimated. This relationship is clearly not the most convenient estimation model, as two estimated functions are nested in one equation. Gonzales and $\mathrm{Wu}(1999)$ state that "Estimation of the value function and weighting function in the context of utility function theory presents challenging problems. A major stumbling block is the need to use the inverse of the value function in estimation. In an experiment, however, one observes the ce rather than $\mathrm{v}(c e)$." They add that due to the model adopted "the standard nonlinear regression technique does not permit an examination of residuals for $v$ and $w$ separately". This means that the Prospect Theory model makes it difficult, if not impossible, to notice any asymmetry of regression errors. Almost every derivation of this model encountered in the literature therefore uses the least squares procedure and assumes a normal error distribution ${ }^{6}$. This, however, may be a very incorrect assumption, as distributions of certainty equivalents are highly skewed, at least on the group level (Kontek, 2010a, 2010b).

In order to avoid these inconvenient characteristics of the model, Gonzales and $\mathrm{Wu}$ (1999) proposed a nonparametric estimation of the Cumulative Prospect Theory model which is, however, very complex and relies on multiple, recursive interpolations of the $\mathrm{v}$ and $\mathrm{W}$ functions, and which, even in the case of two-outcome lotteries, is much more involved than the standard nonlinear least squares procedure.

The relationship to be resolved by the estimation procedure becomes even more complex for three-outcome lotteries (cf. (4.2)) and this may justify limiting Cumulative Prospect Theory to two-outcome lotteries.

5.3. Another reason that the estimations of the Cumulative Prospect Theory model are not widely known might be simply that this model does not work correctly for multiple outcomes. The illustrative example presented in Point 4 may warrant such a conclusion.

As the applicability of Cumulative Prospect Theory to multi-outcome lotteries has not been confirmed by its authors and proponents for so long time, the author of this paper believes it is not his role to undertake this job in the present paper.

5.4. Estimation procedures for the relative utility model in case of two-outcome lotteries have been already presented in former papers by Kontek (2010a, 2010b). To the best of the author's knowledge, this was the widest coverage of estimation methods for lottery experiments ever undertaken.

\footnotetext{
${ }^{6}$ The maximum likelihood estimations for two-outcome lotteries which are encountered in the literature assume a normal error distribution as well. This reduces the methodology to the least squares procedure.
} 
One group of methods concerned standard methods: Mean (Least Squares), Quantile (including Median) and Mode regression procedures performed both parametrically and nonparametrically. However, the standard median and (especially) mode estimators are characterized by computational inconveniences, which may lead to difficulties in finding the global optimum. A novel approach has therefore been proposed, one based on the densities of certainty equivalents (Kontek, 2010b). Despite presenting this very promising methodology, the present paper concentrates on standard regression techniques. This is because the use of more advanced methods would unnecessarily complicate this text and convey the impression that the derivation of the relative utility model is a highly sophisticated subject. The use of density based regression methods for multi-outcome lotteries will therefore be presented in a future paper.

5.5. The relative utility model has been estimated using the experimental data presented by Traub and Schmidt (2009) ${ }^{7}$. Their research concerned the relationship between WTP (Willingness to Pay) and WTA (Willingness to Accept). Twenty four subjects participated in the experiment. The data set contains both two- and three-outcome lotteries (25 and 29 respectively), which allows the obtained results to be compared. Only that subset which concerns certainty equivalents was used in the present research.

The experimental values of the certainty equivalents were first transposed to the $[0,1]$ interval using (3.2). The obtained relative certainty equivalents were then checked for consistency. Values of $r$ that were less than 0 or greater than $1^{8}$ were corrected to 0 and 1 respectively, rather than being excluded from the research. The data were otherwise not interfered with.

5.6. It is possible to propose several functional forms for the relative utility function Q. Beta distribution is the only one used in this paper, as it is the best known and most widely used distribution defined over the interval $[0,1]$. Hence, the function $Q$ is described using Cumulative Beta Distribution as follows:

$$
p=\mathrm{Q}(r)=\mathrm{I}(r ; \alpha, \beta),
$$

where I denotes the regularized incomplete beta function. The inverse form of (5.2) is:

$$
r=\mathrm{Q}^{-1}(p)=\mathrm{I}^{-1}(p ; \alpha, \beta),
$$

where $\mathrm{I}^{-1}$ denotes the inverse of the regularized incomplete beta function.

\footnotetext{
${ }^{7}$ Their data have been used by Hey, Morone and Schmidt (2009) to derive the Expected Utility and RankDependent Expected Utility models. The reader is encouraged to compare the methodologies and results.

${ }^{8}$ These resulted from providing certainty equivalent values outside the acceptable range (e.g. $\$ 25$ for a $\$ 30-40$ lottery or $\$ 12$ for a $\$ 0$-10 lottery).
} 
5.7. The general regression model with additive errors is written in vector notation as (Cameron, Trivedi, 2005):

$$
y=E[y \mid x]+e
$$

where $\mathrm{E}[y \mid x]$ denotes the conditional expectation of the observed variable $y$ given explanatory variable $x$, and $e$ denotes a vector of unobserved random errors. The nonlinear estimation model assumes $\mathrm{E}[y \mid x]$ to be a nonlinear function $g(x ; \theta)$, where $\theta$ denotes a vector of parameters. The error term is then defined as the difference between the observed variable and its conditional expectation, $e_{i}=y_{i}-g\left(x_{i} ; \theta\right)$. The loss function $L(e)$ defines the loss associated with the error $e$. The estimation procedure minimizes the expected value of the loss function (expected loss), and importantly, the type of the loss function determines the conditional expectation of the result.

5.8. If the loss function is the square of the error, then the conditional expectation of $y$ is the mean of $x$. In the case of two-outcome lotteries, the least squares (mean) regression estimator minimizes the following function (cf. (3.4)):

$$
\mathrm{S}_{\text {mean }}(\theta)=\sum_{i=1}^{N}\left[r_{i}-\mathrm{Q}^{-1}\left(p_{i} ; \theta\right)\right]^{2}
$$

where $i$ denotes the next among all $N$ data. In case of more than two outcomes, the least squares (mean) estimator minimizes the following function (cf. (3.6)):

$$
\mathrm{S}_{\text {mean }}(\theta)=\sum_{i=1}^{N}\left[r_{i}-\mathrm{Q}^{-1}\left(\sum_{i=1}^{n} p_{i} \mathrm{Q}\left(r_{i} ; \theta\right) ; \theta\right)\right]^{2}
$$

Clearly, (5.6) is more general than (5.5), and may be used for two-outcome lotteries as well. Please note that the estimated function Q and its parameters $\theta$ appear twice in (5.6). They are first used to calculate the equivalent probability $p_{e q}$ (cf. (3.5)) and then to convert this probability to the relative certainty equivalent (cf. (3.4)).

Assuming that function Q has $k$ parameters, the number of degrees of freedom is $N-$ $k$. It follows that the average estimation error is given by:

$$
e r r_{\text {mean }}=\sqrt{\frac{\operatorname{Min}\left(S_{\text {mean }}\right)}{N-k}}
$$

The results of the least squares regression procedure are presented in Figure 5.1. As shown, the function for three-outcome lotteries is only slightly more curved than that for twooutcome lotteries. The estimation errors are about $10 \%$ lower in the case of three-outcome lotteries, which is a somewhat surprising result. 


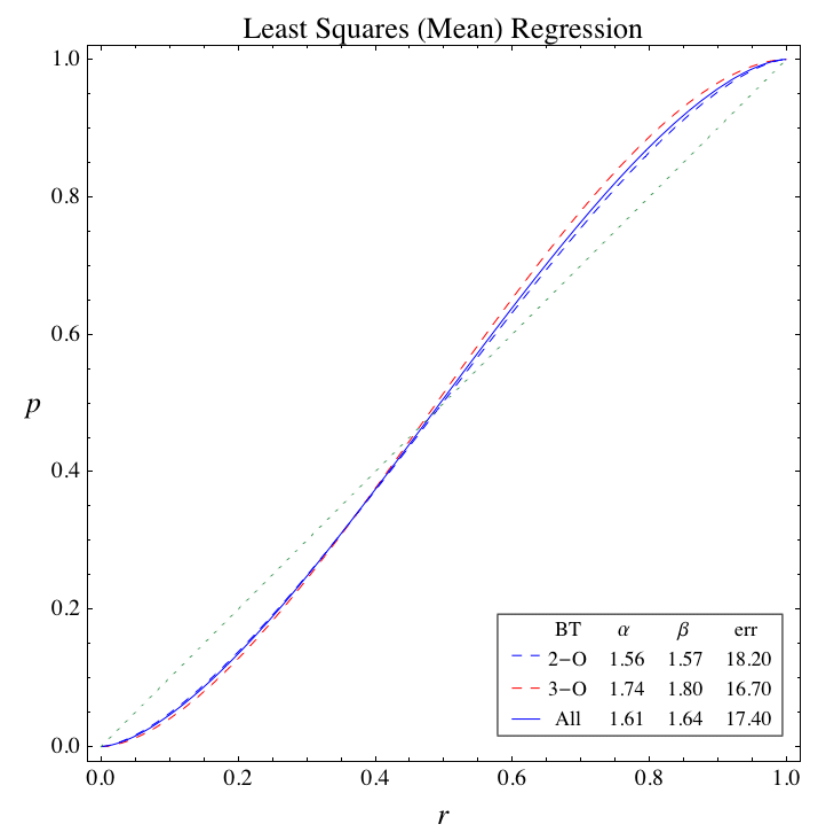

Figure 5.1. Estimation results for the least squares (mean) regression. Estimation for two-outcome lotteries are marked with a blue dashed line, for three-outcome lotteries with a red dashed line, and for all lotteries with a blue solid line. Errors are given as percentages.

5.9. If the loss function is absolute error, then the conditional expectation of $y$ is the median of $x$. In the case of two-outcome lotteries, the median regression estimator minimizes:

$$
\mathrm{S}_{\mathrm{med}}(\theta)=\sum_{i=1}^{N}\left|r_{i}-\mathrm{Q}^{-1}\left(p_{i} ; \theta\right)\right|
$$

In case of more than two outcomes the median estimator minimizes:

$$
\mathrm{S}_{\text {med }}(\theta)=\sum_{i=1}^{N}\left|r_{i}-\mathrm{Q}^{-1}\left(\sum_{i=1}^{n} p_{i} \mathrm{Q}\left(r_{i} ; \theta\right) ; \theta\right)\right|
$$

Equation (5.9) is more general than (5.8), and works for two-outcome lotteries as well. As with the least squares regression procedure, the estimated function $\mathrm{Q}$ and its parameters $\theta$ appear twice in (5.9).

The average absolute error is defined as:

$$
e r r_{m e d}=\frac{\operatorname{Min}\left(S_{m e d}\right)}{N-k}
$$

The median regression estimations are presented in Figure 5.2. Very clearly all median regression procedures gave the same result, which is a straight line. This means that lottery valuations are equal to their expected values. 


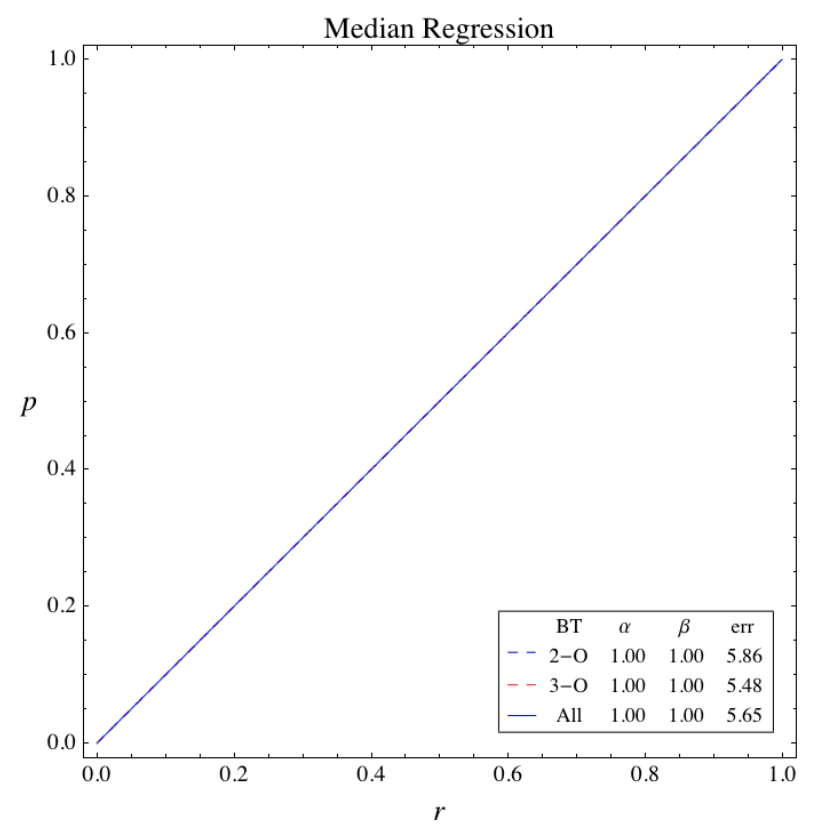

Figure 5.2. Estimation results for the median regression. Estimation for two-outcome lotteries, threeoutcome lotteries, and for all lotteries are the same. Errors are given as percentages.

5.10. If the loss function is asymmetric absolute error with a penalty of $q|e|$ on underprediction and a penalty of $(1-q)|e|$ on overprediction, then the conditional expectation of $y$ is the $q$ th quantile of $x$. In the case of two-outcome lotteries, the $q$ th quantile regression estimator minimizes:

$$
\mathrm{S}_{\mathrm{q}}(\theta)=\sum_{i=1}^{N} q\left[r_{i}-\mathrm{Q}^{-1}\left(p_{i} ; \theta\right)\right]^{+}+\sum_{i=1}^{N}(1-q)\left[r_{i}-\mathrm{Q}^{-1}\left(p_{i} ; \theta\right)\right]^{-}
$$

where a shorthand notation is used: $[a]^{+}=a$ for positive $a$, and 0 for negative $a$; similarly $[a]^{-}$ $=-a$ for negative $a$, and 0 for positive $a$. In the special case of $q=0.5,(5.11)$ reduces to (5.8). For lotteries with more than two outcomes the quantile estimator minimizes:

$\mathrm{S}_{\mathrm{q}}(\theta)=\sum_{i=1}^{N} q\left[r_{i}-\mathrm{Q}^{-1}\left(\sum_{i=1}^{n} p_{i} \mathrm{Q}\left(r_{i} ; \theta_{\text {med }}\right) ; \theta\right)\right]^{+}+\sum_{i=1}^{N}(1-q)\left[r_{i}-\mathrm{Q}^{-1}\left(\sum_{i=1}^{n} p_{i} \mathrm{Q}\left(r_{i} ; \theta_{\text {med }}\right) ; \theta\right)\right]^{-}$

The relative utility function Q appears twice in (5.12) but with different parameters. Calculation of the equivalent probability is done using parameters $\theta_{\text {med }}$ obtained in the median regression procedure. Calculation of the relative certainty equivalent is done using the sought $\theta$ parameters.

The interpretation of the value $\mathrm{S}_{\mathrm{q}}$ obtained by minimizing (5.11) or (5.12) may not be as clear as for median estimation, but (5.10) may be kept for convenience.

The results of the quantile estimator are presented in Figure 5.3. In the case of lower quartile regression, there is a slight difference between two- and three-outcome lotteries. 
However upper quartile regression estimations are essentially the same. Similarly to previously presented estimations, the estimation errors are lower in case of three-outcome lotteries.
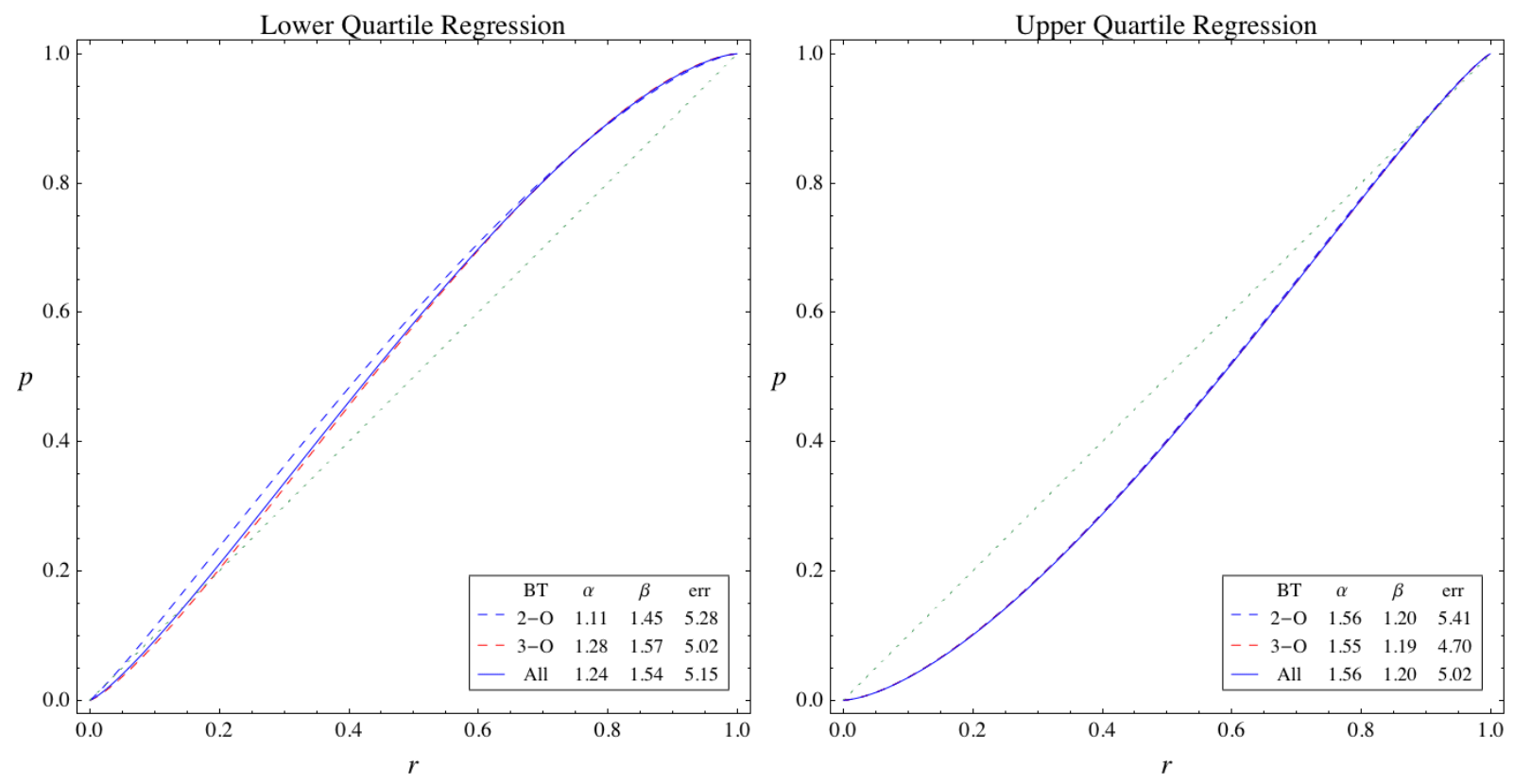

Figure 5.3. Estimation results for the lower quartile (left) and upper quartile (right) regression. Estimation for two-outcome lotteries are marked with a blue dashed line, for three-outcome lotteries with a red dashed line, and for all lotteries with a blue solid line. Errors are given as percentages.

\section{Representation of Lotteries}

6.1. It is always convenient to observe the data points and estimated curve on one graph. This, however, is rarely possible in regression estimation as models typically use more than one explanatory variable and/or consist of more than one regression function. This is precisely the case with Cumulative Prospect Theory. This theory is a two-equation model and the result is a combination of both functions. Cumulative Prospect Theory additionally uses several explanatory variables. For a lottery with $n$ outcomes, the number of considered probabilities is $n-1$ (as the probabilities sum to 1 , one probability may be rejected from consideration). Therefore a space with $n+(n-1)+1=2 n$ dimensions is needed to present the lottery together with its certainty equivalent. Even in the simplest case of two outcomes, four dimensions are needed (lower outcome, higher outcome, probability of winning one of the outcomes, and certainty equivalent). For both the reasons discussed in this sub-point, lotteries together with estimation results cannot be presented on the one graph using the Cumulative Prospect Theory model.

6.2. The situation, however, is quite the opposite in the case of the relative utility model. Each two-outcome lottery is described by only using two values: the probability of winning the lottery and the relative certainty equivalent. This is because the certainty equiva- 
lent, lower outcome and higher outcome are reduced to one value i.e. the relative certainty equivalent using (3.2). This means there are only two dimensions needed to present a lottery and its certainty equivalent. This enables all two-outcome lotteries from the considered data set to be presented in Figure 6.1

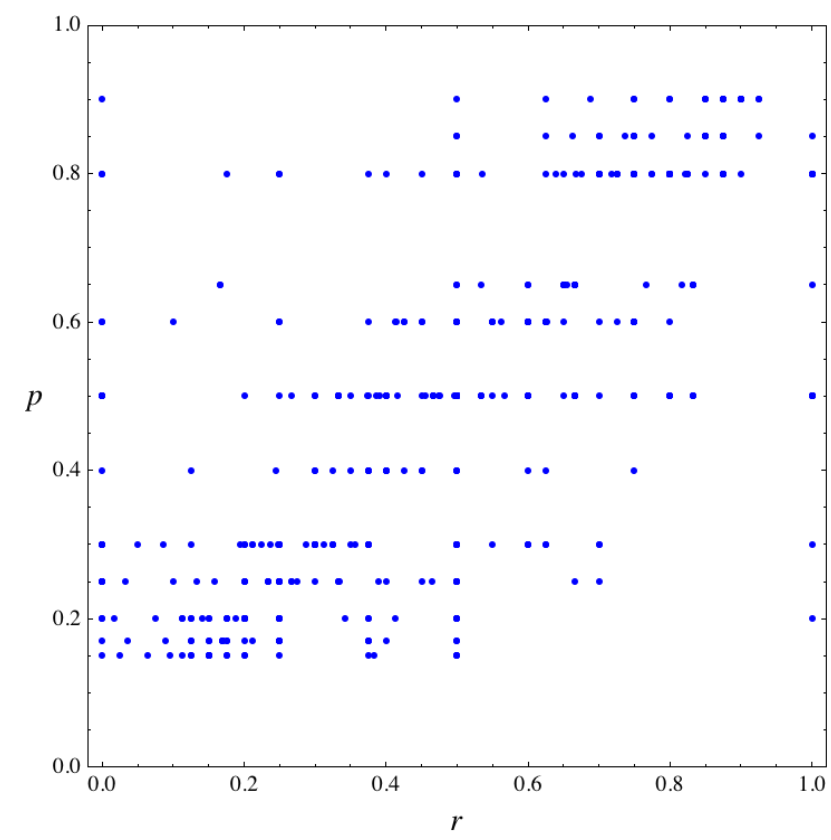

Figure 6.1. Two-outcome lotteries represented as single points on $p-r$ graph.

6.3. Fortunately, the number of dimensions needed to present lotteries with more than two outcomes remains the same. This because each multi-outcome lottery may be represented as a two-outcome lottery according to the relative utility model. As a result, each multioutcome lottery is described by its equivalent probability $p_{\text {eq }}$ defined by (3.5), and by the relative certainty equivalent defined by (3.2). This means that a lottery with any number of outcomes, together with its relative certainty equivalent, only requires two dimensions to be presented. This enables all three-outcome lotteries together with two-outcome lotteries to be presented on a single graph as shown in Figure 6.2.

Multi-outcome lotteries are presented as red points. Please note their location on both graphs is not the same and depends on the relative utility function parameters. The left diagram uses model parameters obtained from the least squares estimation, whereas the right diagram uses parameters obtained from the median estimation. This difference should not come as a surprise, as the perception of multi-outcome lotteries in regard to two-outcome lotteries should depend on people's preferences, whereas these preferences are expressed by the relative utility function. 

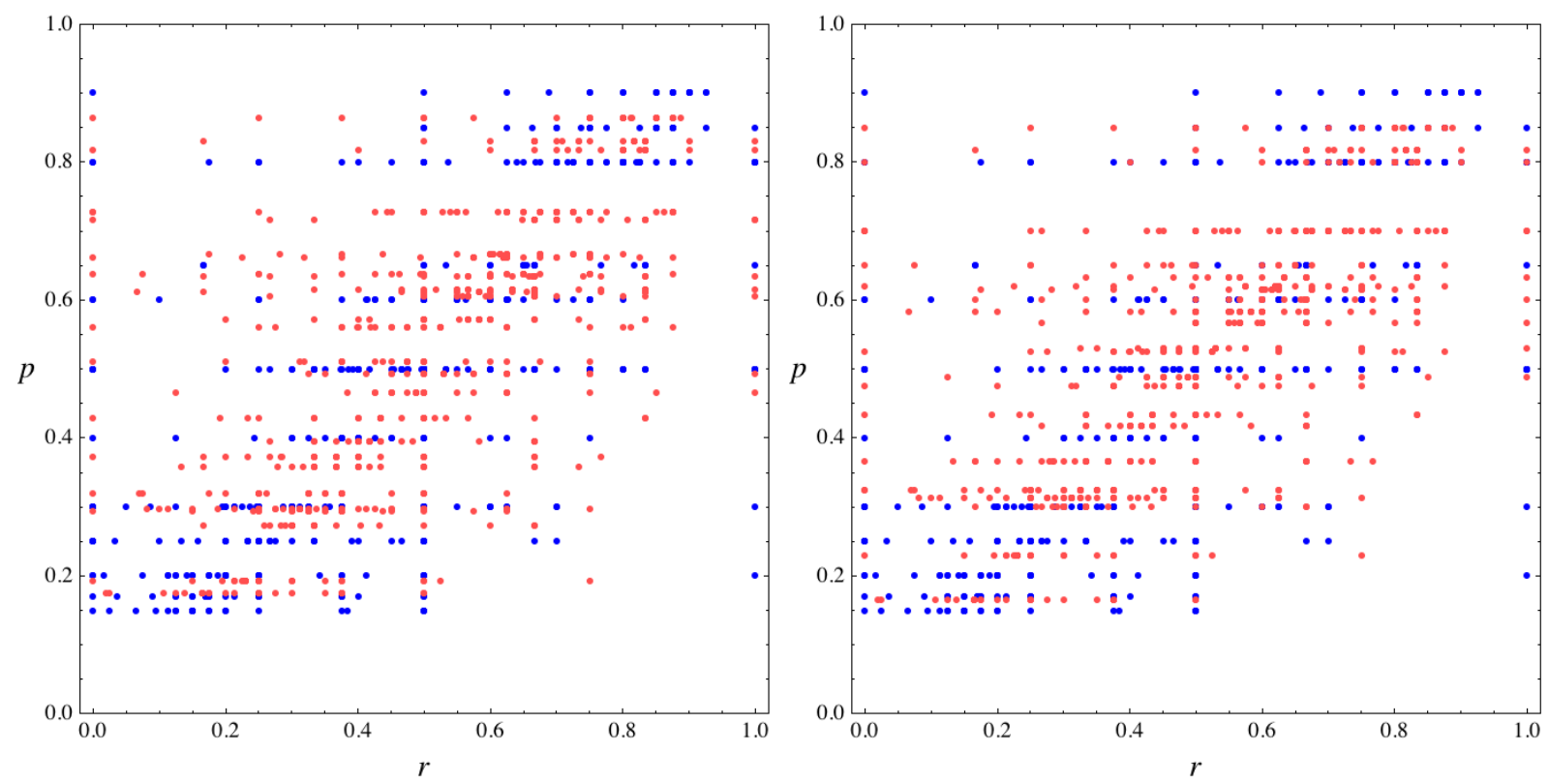

Figure 6.2. Two- and three-outcome lotteries represented as single points on the $p$ - $r$ graph. Twooutcome lotteries are marked with blue points and three-outcome lotteries with red. The three-outcome lotteries represented using the least squares regression parameters are shown in the diagram on the left and those using the median regression parameters on the right.

6.3. As the relative utility is a single-equation and single-variable model, both lotteries and regression estimations may be presented on as a single $p-r$ graph (Figure 6.3).

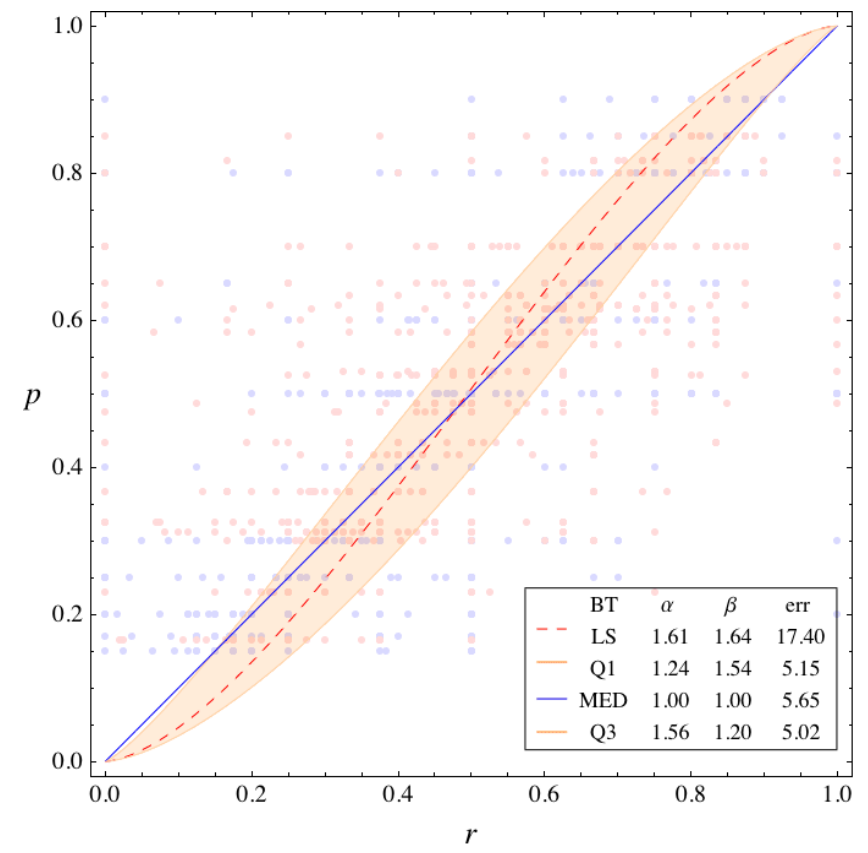

Figure 6.3. Lotteries and regression estimations presented on a single graph. Two-outcome lotteries are marked with light blue points and three-outcome lotteries with light red points. Parameterization from the median regression is used to represent three-outcome lotteries. The least squares (mean) regression estimation is drawn using the red dashed line and the median regression estimation with the solid blue line. The orange shading marks the area between the lower and upper quartiles.

The relative utility model thus offers an additional and important advantage over $\mathrm{Cu}$ mulative Prospect Theory. 


\section{Summary}

7.1. This paper presented two approaches for the analysis of multi-outcome lotteries: the first uses Prospect Theory and the second uses Relative Utility Function. The paper presented several important features of the relative utility approach and demonstrated its advantages over Cumulative Prospect Theory.

The relative utility function strongly resembles the utility function hypothesized by Markowitz (1952). It follows Expected Utility Theory with a transformed outcome domain. It is a simpler, single-equation, model, which describes the experimental results without having recourse to the probability weighting function. It also follows a classical econometric approach when building a regression model, which allows a number of methods to be used for parameters estimation. When used in a hypothetical three-outcome lottery, it gave very sound prediction of people's valuations, and this prediction was consistent with two-outcome lottery results. When used with real data it gave very consistent results for two- and three-outcome lotteries, thus confirming not only correctness of the model but also vindicating the homogeneity of the answers given by subjects. Besides, the model allowed the multi-outcome lotteries, together with the estimation results, to be presented on a single graph.

This paper shows that the relative utility model is superior to Cumulative Prospect Theory in virtually every respect with regard to multi-outcome lotteries. In fact, it is difficult to find any advantages of the Cumulative Prospect Theory model. All that springs to mind is that a paper supporting Cumulative Prospect Theory has a far greater probability of being accepted by a journal than one supporting the relative utility model and this makes this theory still so attractive for further research.

\section{References}

1. Cameron, A. C., Trivedi, P. K., (2005). Microeconometrics. Methods and Applications, Cambridge University Press.

2. Edwards W., (1961). Behavioral Decision Theory. Ann. Rev. Psych., 12, pp 473-479.

3. Gonzales, R., Wu, G., (1999). On the Shape of the Probability Weighting Function, Cognitive Psychology, 38, pp 129-166.

4. Hey, J. D., Morone, A., Schmidt U., (2009). Noise and bias in eliciting preferences, Journal of Risk and Uncertainty, 39, pp 213-235.

5. Kahneman, D., Tversky, A., (1979). Prospect theory: An analysis of decisions under risk. Econometrica, 47, pp 313-327.

6. Kontek, K., (2009a). On Mental Transformations. MPRA Paper http://mpra.ub.unimuenchen.de/16516/, Available at SSRN: http://ssrn.com/abstract=1437722 . 
7. Kontek, K., (2009b). Lottery Valuation Using the Aspiration / Relative Utility Function, Warsaw School of Economics, Department of Applied Econometrics Working Paper no. 39. Available at SSRN: http://ssrn.com/abstract=1437420 and RePec:wse:wpaper:39.

8. Kontek, K., (2010a). Mean, median or Mode? A Striking Conclusion from Lottery Experiments, MPRA Paper http://mpra.ub.uni-muenchen.de/21758/, Available at SSRN:

http://ssrn.com/abstract $=1581436$

9. Kontek, K., (2010b). Density Based Regression for Inhomogeneous Data; Application to Lottery Experiments, MPRA Paper http://mpra.ub.uni-muenchen.de/22268/, Available at SSRN:

http://ssrn.com/abstract $=1593766$

10. Markowitz H., (1952A). The Utility of Wealth. Journal of Political Economy, Vol. 60, 151-158.

11. Quiggin J., (1982). A theory of anticipated utility. Journal of Economic Behavior and Organization 3(4), 323-43.

12. Traub, S., Schmidt, U., (2009). An Experimental Investigation of the Disparity between WTA and WTP for Lotteries, Theory \& Decision, 66, pp 229-262.

13. Tversky A., Kahneman D., (1992). Advances in Prospect Theory: Cumulative Representation of Uncertainty. Journal of Risk and Uncertainty, vol. 5(4), October, 297-323. 